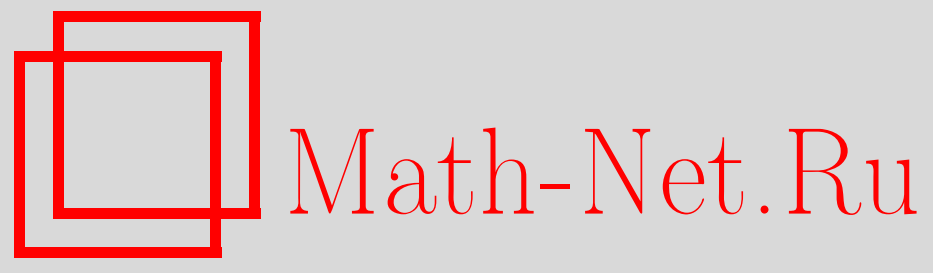

Н. В. Зайцева, Нелокальная краевая задача для $B$ гиперболического уравнения в прямоугольной области, Вестн. Сам. гос. техн. ун-та. Сер. Физ.мат. науки, 2016, номер 4, 589-602

DOI: https://doi.org/10.14498/vsgtu1501

Использование Общероссийского математического портала MathNet.Ru подразумевает, что вы прочитали и согласны с пользовательским соглашением

http://www . mathnet.ru/rus/agreement

Параметры загрузки:

IP : 54.80 .97 .219

26 апреля 2023 г., 14:09:24

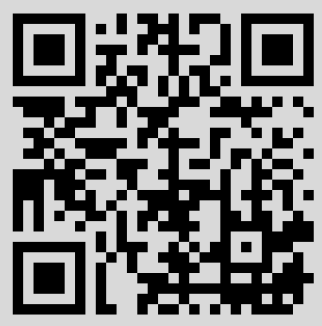


УДК 517.956.3

\title{
НЕЛОКАЛЬНАЯ КРАЕВАЯ ЗАДАЧА ДЛЯ В-ГИПЕРБОЛИЧЕСКОГО УРАВНЕНИЯ В ПРЯМОУГОЛЬНОЙ ОБЛАСТИ
}

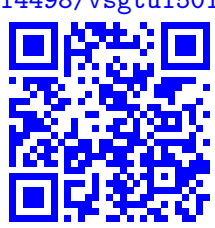

\section{H. В. Зайщева}

Казанский (Приволжский) федеральный университет, Россия, 420008, Казань, ул. Кремлевская, 18.

\begin{abstract}
Аннотация
Для гиперболического уравнения с оператором Бесселя поставлена начально-граничная задача с интегральным нелокальным условием первого рода в прямоугольной области. Поставленная задача с нелокальным интегральным условием первого рода эквивалентно сведена к локальной начально-граничной задаче со смешанными краевыми условиями первого и третьего рода. Методом спектрального анализа доказаны теоремы единственности и существования решения эквивалентной задачи. Решение построено в явном виде в виде ряда Фурье-Бесселя и приведено обоснование сходимости ряда в классе регулярных решений. Доказательство единственности решения эквивалентной задачи проводится на основании полноты системы собственных функций соответствующей одномерной задачи на собственные значения в пространстве квадратично суммируемых функций с весом. Для доказательства существования решения эквивалентной задачи используются оценки коэффициентов ряда и системы собственных функций, которые установлены на основании асимптотических формул для функции Бесселя первого рода при больших значениях аргумента и нулей этой функции. Получены достаточные условия относительно начальных условий, которые гарантируют сходимость построенного ряда в классе регулярных решений. Показана однозначная разрешимость первоначальной задачи.
\end{abstract}

Ключевые слова: гиперболическое уравнение, сингулярный коэффициент, нелокальное интегральное условие, единственность, существование, ряд Фурье--Бесселя, равномерная сходимость.

1. Постановка задачи. Рассмотрим гиперболическое уравнение с оператором Бесселя

$$
\square_{B} u(x, t) \equiv u_{t t}-x^{-k} \frac{\partial}{\partial x}\left(x^{k} \frac{\partial u}{\partial x}\right)=0,
$$

где $k \leqslant-1$ - заданное действительное число, в прямоугольной области

$$
D=\{(x, t) \mid 0<x<l, 0<t<T\},
$$

(C) 2016 Самарский государственный технический университет.

\section{Образец для цитирования}

Зай це в а Н. В. Нелокальная краевая задача для В-гиперболического уравнения в прямоугольной области // Вестн. Сам. гос. техн. ун-та. Сер. Физ.-мат. науки, 2016. Т. 20, № 4. C. 589-602. doi: 10.14498/vsgtu1501.

\section{Сведения об авторе}

Наталъя Владимировна Зайцева (n.v.zaiceva@yandex.ru), ассистент, каф. высшей математики и математического моделирования. 
где $l, T>0$ - заданные действительные числа. Обозначим боковые стороны прямоугольника $D$ через

$$
\Gamma_{0}=\{(x, t) \mid t=0,0 \leqslant x \leqslant l\}, \quad \Gamma_{l}=\{(x, t) \mid x=l, 0 \leqslant t \leqslant T\} .
$$

По аналогии с [1] уравнение (1) будем называть $B$-гиперболическим уравнением. В работе [2, с. 164-225] изучены краевые задачи Коши и КошиГурса для уравнения (1) при $k \geqslant 1$. В работе [3] показана некорректность постановки таких задач при $k<0$. Работы [4,5] посвящены изучению задачи Трикоми для уравнения смешанного типа, у которого гиперболическая часть совпадает с уравнением (1).

В данной работе для уравнения (1) в области $D$ исследуем следующую начально-граничную задачу с нелокальным граничным условием первого рода.

НЕЛОКАЛЬНАЯ ЗАДАЧА. Найти функиию $u(x, t)$, которая удовлетворяет следУющим условиям:

$$
\begin{gathered}
u(x, t) \in C(\bar{D}) \cap C^{1}\left(D \cup \Gamma_{0} \cup \Gamma_{l}\right) \cap C^{2}(D), \\
\square_{B} u(x, t) \equiv 0, \quad(x, t) \in D, \\
u(x, 0)=\varphi(x), \quad u_{t}(x, 0)=\psi(x), \quad 0 \leqslant x \leqslant l, \\
u(0, t)=0, \quad 0 \leqslant t \leqslant T, \\
\int_{0}^{l} u(x, t) x d x=A=\text { const, } \quad 0 \leqslant t \leqslant T,
\end{gathered}
$$

где $A$ - заданное число, $\varphi(x), \psi(x)$ - заданнье достаточно гладкие функиии, удовлетворяющие условиям согласования

$$
\int_{0}^{l} \varphi(x) x d x=A, \quad \int_{0}^{l} \psi(x) x d x=0 .
$$

В поставленной задаче (2)-(7) граничное условие (6) является нелокальным. Интегральное условие такого типа ранее возникло в работах [6-8] для уравнения теплопроводности, например, в [8] при изучении вопроса об устойчивости разрежения плазмы. Физически нелокальное условие (6) означает постоянство внутренней энергии системы. Термин «интегральное условие первого рода» был введен в работе [9].

Нелокальные задачи с интегральными условиями для гиперболических уравнений изучаются с начала 90-х годов прошлого столетия и до сих пор вызывают большой интерес у математиков, так как ряд процессов, изучаемых в физике, химии, биологии, нередко приводит к постановкам задач с такими нелокальными условиями. Этим и обусловлено появление в математической литературе за последние десятилетия большого количества публикаций в этом направлении.

В работах $[10,11]$ впервые методами функционального анализа изучены краевые задачи с интегральными условиями типа (6) и более сложными условиями для уравнения (1) при $k=0$, для телеграфного уравнения и для более общих уравнений гиперболического типа с гладкими коэффициентами вида

$$
u_{t t}-\left(a(x, t) u_{x}\right)_{x}+c(x, t) u=f(x, t) .
$$


Исследованию нелокальных задач для уравнений с оператором Бесселя посвящены работы [12-15]. В работах [16-19] впервые исследованы краевые задачи для уравнений смешанного типа в прямоугольной области с нелокальным интегральным условием типа (6).

В данной работе методом спектрального анализа [16-19] доказаны теоремы единственности и существования решения задачи (2)-(7) при всех $k \leqslant-1$. При этом решение построено в виде суммы ряда Фурье-Бесселя и приведено обоснование сходимости ряда в классе регулярных решений (2) и (3). Нами также был изучен случай, когда $k \geqslant 1$ [20], но уже с другими граничными условиями.

Умножим уравнение (1) на $x$ и проинтегрируем при фиксированном $t \in$ $(0, T)$ по переменной $x$ на промежутке от $\varepsilon$ до $l-\varepsilon$, где $\varepsilon>0$ - достаточно малое число. В результате получим

$$
\int_{\varepsilon}^{l-\varepsilon} u_{t t} x d x-\int_{\varepsilon}^{l-\varepsilon}\left(x u_{x x}+k u_{x}\right) d x=0,
$$

или

$$
\frac{d^{2}}{d t^{2}} \int_{\varepsilon}^{l-\varepsilon} u(x, t) x d x-\int_{\varepsilon}^{l-\varepsilon} \frac{\partial}{\partial x}\left(x u_{x}+(k-1) u\right) d x=0 .
$$

Отсюда будем иметь

$$
\frac{d^{2}}{d t^{2}} \int_{\varepsilon}^{l-\varepsilon} u(x, t) x d x-\left.\left(x u_{x}+(k-1) u\right)\right|_{\varepsilon} ^{l-\varepsilon}=0
$$

В силу условий (2), (5) и (6) в последнем равенстве можно перейти к пределу при $\varepsilon \rightarrow 0$, в результате чего получим граничное условие третьего рода

$$
l u_{x}(l, t)+(k-1) u(l, t)=0, \quad 0 \leqslant t \leqslant T .
$$

В дальнейшем будем рассматривать задачу (2)-(5), (8).

2. Единственность решения задачи. Частные решения уравнения (1), не равные нулю в области $D$ и удовлетворяющие условиям $(2),(5)$ и $(8)$, будем искать в виде $u(x, t)=X(x) T(t)$. Подставляя данную функцию в уравнение $(1)$ и условия $(5)$ и $(8)$, получим относительно функции $X(x)$ спектральную задачу:

$$
\begin{gathered}
X^{\prime \prime}(x)+\frac{k}{x} X^{\prime}(x)+\lambda^{2} X(x)=0, \quad 0<x<l, \\
X(0)=0, \quad l X^{\prime}(l)+(k-1) X(l)=0,
\end{gathered}
$$

где $\lambda^{2}$ - постоянная разделения.

Общее решение уравнения (9) при $k \leqslant-1$ определяется формулой

$$
X(x)=C_{1} x^{\frac{1-k}{2}} J_{\frac{1-k}{2}}(\lambda x)+C_{2} x^{\frac{1-k}{2}} Y_{\frac{1-k}{2}}(\lambda x)
$$

где $J_{\nu}(\xi), Y_{\nu}(\xi)$ - функции Бесселя первого и второго рода, соответственно, порядка $\nu=(1-k) / 2 ; C_{1}, C_{2}$ - произвольные постоянные. 
Для того чтобы функция (11) удовлетворяла первому условию из (10), необходимо положить значения констант $C_{1}=1$ и $C_{2}=0$. В результате данное решение принимает вид

$$
X(x)=x^{\frac{1-k}{2}} J_{\frac{1-k}{2}}(\lambda x) .
$$

Теперь подставим функцию (12) во второе граничное условие из (10) и получим уравнение

$$
\mu J_{\nu}^{\prime}(\mu)-\nu J_{\nu}(\mu)=0, \quad \mu=\lambda l, \quad \nu=\frac{1-k}{2} .
$$

В силу теоремы 2 [19, с. 310] уравнение (13) при $\nu>-1$ имеет лишь вещественные простые нули. С другой стороны, на основании формулы [19, c. 305]

$$
z J_{\nu}^{\prime}(z)-\nu J_{\nu}(z)=-z J_{\nu+1}(z)
$$

уравнение (13) равносильно уравнению

$$
J_{\frac{3-k}{2}}(\mu)=0, \quad \mu=\lambda l .
$$

Для нулей уравнения (14), согласно [21, с. 317], при больших $n$ справедлива асимптотическая формула

$$
\mu_{n}=\lambda_{n} l=\pi n+\frac{\pi}{2}-\frac{\pi}{4} k+O\left(\frac{1}{n}\right)
$$

Таким образом, система собственных функций задачи (9) и (10) имеет вид

$$
X_{n}(x)=x^{\frac{1-k}{2}} J_{\frac{1-k}{2}}\left(\frac{\mu_{n} x}{l}\right)=x^{\frac{1-k}{2}} J_{\frac{1-k}{2}}\left(\lambda_{n} x\right), \quad n \in \mathbb{N},
$$

а собственные значения $\mu_{n}$ определяются как нули уравнения (14).

Известно [19, с. 308-315], что система собственных функций (16) ортогональна и полна в пространстве $L_{2}[0, l]$ с весом $x^{k}$.

Далее, следуя [22,23], рассмотрим функции

$$
u_{n}(t)=\int_{0}^{l} u(x, t) x^{k} X_{n}(x) d x, \quad n=1,2, \ldots,
$$

где $X_{n}(x)$ определяются по формуле (16). На основании (17) введем в рассмотрение вспомогательные функции вида

$$
u_{n, \varepsilon}(t)=\int_{\varepsilon}^{l-\varepsilon} u(x, t) x^{k} X_{n}(x) d x, \quad n=1,2, \ldots,
$$

где $\varepsilon>0$ - достаточно малое число. Продифференцируем равенство (18) по переменной $t$ дважды при $0<t<T$ и с учетом уравнения (1) получим

$$
u_{n, \varepsilon}^{\prime \prime}(t)=\int_{\varepsilon}^{l-\varepsilon} u_{t t}(x, t) x^{k} X_{n}(x) d x=\int_{\varepsilon}^{l-\varepsilon}\left(u_{x x}+\frac{k}{x} u_{x}\right) x^{k} X_{n}(x) d x=
$$




$$
=\int_{\varepsilon}^{l-\varepsilon} \frac{\partial}{\partial x}\left(x^{k} u_{x}\right) X_{n}(x) d x=\left.x^{k} u_{x} X_{n}(x)\right|_{\varepsilon} ^{l-\varepsilon}-\int_{\varepsilon}^{l-\varepsilon} x^{k} u_{x} X_{n}^{\prime}(x) d x
$$

Из (18) в силу уравнения (9) будем иметь

$$
\begin{gathered}
u_{n, \varepsilon}(t)=-\frac{1}{\lambda_{n}^{2}} \int_{\varepsilon}^{l-\varepsilon} u(x, t) x^{k}\left[X_{n}^{\prime \prime}(x)+\frac{k}{x} X_{n}^{\prime}(x)\right] d x= \\
=-\frac{1}{\lambda_{n}^{2}} \int_{\varepsilon}^{l-\varepsilon} u(x, t) \frac{d}{d x}\left(x^{k} X_{n}^{\prime}(x)\right) d x= \\
=-\frac{1}{\lambda_{n}^{2}}\left[\left.u(x, t) x^{k} X_{n}^{\prime}(x)\right|_{\varepsilon} ^{l-\varepsilon}-\int_{\varepsilon}^{l-\varepsilon} x^{k} u_{x} X_{n}^{\prime}(x) d x\right],
\end{gathered}
$$

откуда находим

$$
\int_{\varepsilon}^{l-\varepsilon} x^{k} u_{x} X_{n}^{\prime}(x) d x=\lambda_{n}^{2} u_{n, \varepsilon}(t)+\left.u(x, t) x^{k} X_{n}^{\prime}(x)\right|_{\varepsilon} ^{l-\varepsilon} .
$$

Подставляя (20) в (19), будем иметь

$$
u_{n, \varepsilon}^{\prime \prime}(t)=\left.x^{k} u_{x} X_{n}(x)\right|_{\varepsilon} ^{l-\varepsilon}-\lambda_{n}^{2} u_{n, \varepsilon}(t)-\left.u(x, t) x^{k} X_{n}^{\prime}(x)\right|_{\varepsilon} ^{l-\varepsilon}
$$

Из формулы (16) следует, что $X_{n}(x)=O\left(x^{1-k}\right)$ и $X_{n}^{\prime}(x)=O\left(x^{-k}\right)$ при $x \rightarrow 0$. И, переходя в $(21)$ к пределу при $\varepsilon \rightarrow 0$, с учетом условий $(2),(5),(8)$ и (10) получим для определения функций $u_{n}(t)$ уравнение

$$
u_{n}^{\prime \prime}(t)+\lambda_{n}^{2} u_{n}(t)=0, \quad t \in(0, T),
$$

общее решение которого имеет вид

$$
u_{n}(t)=a_{n} \cos \lambda_{n} t+b_{n} \sin \lambda_{n} t
$$

где $a_{n}, b_{n}$-произвольные постоянные. Для определения этих постоянных функции (17) удовлетворим начальным условиям (4):

$$
\begin{aligned}
u_{n}(0) & =\int_{0}^{l} u(x, 0) x^{k} X_{n}(x) d x=\int_{0}^{l} \varphi(x) x^{k} X_{n}(x) d x=\varphi_{n}, \\
u_{n}^{\prime}(0) & =\int_{0}^{l} u_{t}(x, 0) x^{k} X_{n}(x) d x=\int_{0}^{l} \psi(x) x^{k} X_{n}(x) d x=\psi_{n} .
\end{aligned}
$$

С учетом (23) и (24) из (22) будем иметь

$$
u_{n}(0)=a_{n}=\varphi_{n}, \quad u_{n}^{\prime}(0)=b_{n} \lambda_{n}=\psi_{n},
$$

откуда находим

$$
a_{n}=\varphi_{n}, \quad b_{n}=\frac{\psi_{n}}{\lambda_{n}}
$$


Подставляя значения (25) в (22), найдем окончательный вид функции

$$
u_{n}(t)=\varphi_{n} \cos \lambda_{n} t+\frac{\psi_{n}}{\lambda_{n}} \sin \lambda_{n} t
$$

Пусть $\varphi(x) \equiv 0$ и $\psi(x) \equiv 0$, тогда из (23) и (24) при всех $n \in \mathbb{N}$ следует, что $\varphi_{n}=\psi_{n} \equiv 0$. Из $(26)$ получим, что $u_{n}(t)=0$ при всех $n \in \mathbb{N}$. Тогда из (17) при любом $t \in[0, T]$ имеем

$$
\int_{0}^{l} u(x, t) x^{k} X_{n}(x) d x=0 .
$$

Отсюда в силу полноты системы (16) в пространстве $L_{2}[0, l]$ с весом $x^{k}$ следует, что $u(x, t)=0$ почти всюду на промежутке $[0, l]$ при любом $t \in[0, T]$. Поскольку, согласно (2), функция $u(x, t) \in C(\bar{D})$, она тождественно равна нулю в $\bar{D}$.

Таким образом, доказана

Теорема 1. Если существует решение задачи (2)-(5), (8), то оно единственно.

3. Существование решения задачи. На основании найденных частных решений (16) и (26) решение задачи (2)-(5), (8) запишем в виде ряда ФурьеБесселя

$$
u(x, t)=\sum_{n=1}^{\infty} u_{n}(t) X_{n}(x),
$$

где функции $u_{n}(t)$ определяются по формуле $(26)$, а функции $X_{n}(x)$ - по формуле (16).

Рассмотрим следующие ряды, полученные формально из ряда (27) почленным дифференцированием:

$$
\begin{aligned}
& u_{t}(x, t)=\sum_{n=1}^{\infty} u_{n}^{\prime}(t) X_{n}(x), \quad u_{x}(x, t)=\sum_{n=1}^{\infty} u_{n}(t) X_{n}^{\prime}(x) . \\
& u_{t t}(x, t)=\sum_{n=1}^{\infty} u_{n}^{\prime \prime}(t) X_{n}(x), \quad u_{x x}(x, t)=\sum_{n=1}^{\infty} u_{n}(t) X_{n}^{\prime \prime}(x) .
\end{aligned}
$$

Теперь покажем, что при определенных условиях относительно функций $\varphi(x)$ и $\psi(x)$, входящих в начальные условия (4) задачи, ряд (27) и ряды (28), (29) сходятся равномерно в области $\bar{D}$.

Лемма 1. Для достаточно больших $n$ и при любом $t \in[0, T]$ справедливъ оценки

$$
\begin{gathered}
\left|u_{n}(t)\right| \leqslant C_{1}\left(\left|\varphi_{n}\right|+\frac{\left|\psi_{n}\right|}{n}\right), \\
\left|u_{n}^{\prime}(t)\right| \leqslant C_{2}\left(n\left|\varphi_{n}\right|+\left|\psi_{n}\right|\right), \\
\left|u_{n}^{\prime \prime}(t)\right| \leqslant C_{3}\left(n^{2}\left|\varphi_{n}\right|+n\left|\psi_{n}\right|\right),
\end{gathered}
$$


где $C_{i}-$ здесъ и далее положительные постоянные.

До каз а т ель ст в о оценок (30)-(32) непосредственно следует из формул (26) и (15).

Лемма 2. Для достаточно больиих $n$ и при всех $x \in[0, l]$ выполнены оценки

$$
\begin{aligned}
& \left|X_{n}(x)\right| \leqslant C_{4} n^{-\frac{1}{2}} \\
& \left|X_{n}^{\prime}(x)\right| \leqslant C_{5} n^{\frac{1}{2}} \\
& \left|X_{n}^{\prime \prime}(x)\right| \leqslant C_{6} n^{\frac{3}{2}}
\end{aligned}
$$

Доказательств о. Функция $X_{n}(x)=x^{\frac{1-k}{2}} J_{\frac{1-k}{2}}\left(\lambda_{n} x\right) \in C^{2}[0, l]$ и при больших $\xi$ справедлива оценка

$$
J_{\nu}(\xi)=O\left(\frac{1}{\xi^{1 / 2}}\right) .
$$

Тогда из (36) следует справедливость оценки (33). Вычислим теперь

$$
X_{n}^{\prime}(x)=\lambda_{n} x^{\frac{1-k}{2}} J_{-\frac{k+1}{2}}\left(\lambda_{n} x\right) .
$$

Тогда из (36) и (37) следует справедливость оценки (34).

Из уравнения (9) запишем

$$
X_{n}^{\prime \prime}(x)=-\frac{k}{x} X_{n}^{\prime}(x)-\lambda_{n}^{2} X_{n}(x) .
$$

Отсюда в силу неравенств (33) и (34) следует справедливость оценки (35).

Согласно леммам 1 и 2 и формуле (15), при любом $(x, t) \in \bar{D}$ ряды $(27)$, (28) и (29) мажорируются соответственно рядами

$$
\begin{gathered}
C_{7} \sum_{n=1}^{\infty}\left(n^{-\frac{1}{2}}\left|\varphi_{n}\right|+n^{-\frac{3}{2}}\left|\psi_{n}\right|\right), \\
C_{8} \sum_{n=1}^{\infty}\left(n^{\frac{1}{2}}\left|\varphi_{n}\right|+n^{-\frac{1}{2}}\left|\psi_{n}\right|\right), \\
C_{9} \sum_{n=1}^{\infty}\left(n^{\frac{3}{2}}\left|\varphi_{n}\right|+n^{\frac{1}{2}}\left|\psi_{n}\right|\right) .
\end{gathered}
$$

Исследуем ряды (38)-(40) на сходимость.

ЛЕмма 3. Если функиии $\varphi(x) \in C^{3}[0, l], \psi(x) \in C^{2}[0, l] u$

$$
\varphi(0)=\varphi(l)=\varphi^{\prime}(l)=\varphi^{\prime \prime}(0)=\varphi^{\prime \prime}(l)=\psi(0)=\psi(l)=\psi^{\prime}(l)=0,
$$

то выполняются оценки

$$
\left|\varphi_{n}\right| \leqslant \frac{C_{10}}{n^{4}}, \quad\left|\psi_{n}\right| \leqslant \frac{C_{11}}{n^{2}} .
$$


Доказательство. С учетом (9), (10) и условий леммы из (23) будем иметь

$$
\begin{gathered}
\varphi_{n}=\int_{0}^{l} \varphi(x) x^{k} X_{n}(x) d x=-\frac{1}{\lambda_{n}^{2}} \int_{0}^{l} \varphi(x)\left(x^{k} X_{n}^{\prime}(x)\right)^{\prime} d x= \\
=-\frac{1}{\lambda_{n}^{2}}\left[\left.\varphi(x) x^{k} X_{n}^{\prime}(x)\right|_{0} ^{l}-\int_{0}^{l} \varphi^{\prime}(x) x^{k} X_{n}^{\prime}(x) d x\right]=\frac{1}{\lambda_{n}^{2}} \int_{0}^{l} \varphi^{\prime}(x) x^{k} X_{n}^{\prime}(x) d x= \\
=\frac{1}{\lambda_{n}^{2}}\left[\left.\varphi^{\prime}(x) x^{k} X_{n}(x)\right|_{0} ^{l}-\int_{0}^{l}\left(\varphi^{\prime}(x) x^{k}\right)^{\prime} X_{n}(x) d x\right]=-\frac{1}{\lambda_{n}^{2}} \int_{0}^{l}\left(\varphi^{\prime}(x) x^{k}\right)^{\prime} X_{n}(x) d x= \\
=-\frac{1}{\lambda_{n}^{2}} \int_{0}^{l} \varphi^{\prime \prime}(x) x^{k} X_{n}(x) d x-\frac{k}{\lambda_{n}^{2}} \int_{0}^{l} \frac{\varphi^{\prime}(x)}{x} x^{k} X_{n}(x) d x .
\end{gathered}
$$

Введем обозначения

$$
\varphi_{n}^{(2)}=\int_{0}^{l} \varphi^{\prime \prime}(x) x^{k} X_{n}(x) d x, \varphi_{1 n}=\int_{0}^{l} \varphi_{1}(x) x^{k} X_{n}(x) d x, \varphi_{1}(x)=\frac{\varphi^{\prime}(x)}{x} .
$$

В результате получим

$$
\varphi_{n}=-\frac{1}{\lambda_{n}^{2}} \varphi_{n}^{(2)}-\frac{k}{\lambda_{n}^{2}} \varphi_{1 n} .
$$

В силу (9) и (10) и условий $\varphi^{\prime \prime}(0)=\varphi^{\prime \prime}(l)=0$ из первого интеграла в (42) получим

$$
\begin{aligned}
& \varphi_{n}^{(2)}=\int_{0}^{l} \varphi^{\prime \prime}(x) x^{k} X_{n}(x) d x=-\frac{1}{\lambda_{n}^{2}} \int_{0}^{l} \varphi^{\prime \prime}(x)\left(x^{k} X_{n}^{\prime}(x)\right)^{\prime} d x= \\
&=-\frac{1}{\lambda_{n}^{2}}\left[\left.\varphi^{\prime \prime}(x) x^{k} X_{n}^{\prime}(x)\right|_{0} ^{l}-\int_{0}^{l} \varphi^{\prime \prime \prime}(x) x^{k} X_{n}^{\prime}(x) d x\right]= \\
&=\frac{1}{\lambda_{n}^{2}} \int_{0}^{l} \varphi^{\prime \prime \prime}(x) x^{k} X_{n}^{\prime}(x) d x=\frac{\varphi_{n}^{(3)}}{\lambda_{n}^{2}},
\end{aligned}
$$

где

$$
\varphi_{n}^{(3)}=\int_{0}^{l} \varphi^{\prime \prime \prime}(x) x^{k} X_{n}^{\prime}(x) d x .
$$

Аналогично на основании $(9),(10)$ и $\varphi(0)=\varphi^{\prime}(l)=0$ проинтегрируем по частям два раза второй интеграл из (42):

$$
\begin{gathered}
\varphi_{1 n}=\int_{0}^{l} \varphi_{1}(x) x^{k} X_{n}(x) d x=-\frac{1}{\lambda_{n}^{2}} \int_{0}^{l} \varphi_{1}(x)\left(x^{k} X_{n}^{\prime}(x)\right)^{\prime} d x= \\
=-\frac{1}{\lambda_{n}^{2}}\left[\left.\varphi_{1}(x) x^{k} X_{n}^{\prime}(x)\right|_{0} ^{l}-\int_{0}^{l} \varphi_{1}^{\prime}(x) x^{k} X_{n}^{\prime}(x) d x\right]= \\
=\frac{1}{\lambda_{n}^{2}} \int_{0}^{l} \varphi_{1}^{\prime}(x) x^{k} X_{n}^{\prime}(x) d x=\frac{\varphi_{1 n}^{(1)}}{\lambda_{n}^{2}},
\end{gathered}
$$


где

$$
\varphi_{1 n}^{(1)}=\int_{0}^{l} \varphi_{1}^{\prime}(x) x^{k} X_{n}^{\prime}(x) d x,
$$

и этот интеграл в силу (37) сходится.

Подставив (44) и (45) в равенство (43), найдем

$$
\varphi_{n}=-\frac{1}{\lambda_{n}^{4}} \varphi_{n}^{(3)}-\frac{k}{\lambda_{n}^{4}} \varphi_{1 n}^{(1)} .
$$

Из (46) следует первая оценка из (41).

На основании $(23)$ при $\psi(0)=\psi(l)=\psi^{\prime}(l)=0$, производя аналогичные вычисления, получим

$$
\begin{gathered}
\psi_{n}=\int_{0}^{l} \psi(x) x^{k} X_{n}(x) d x= \\
=-\frac{1}{\lambda_{n}^{2}} \int_{0}^{l} \psi^{\prime \prime}(x) x^{k} X_{n}(x) d x-\frac{k}{\lambda_{n}^{2}} \int_{0}^{l} \frac{\psi^{\prime}(x)}{x} x^{k} X_{n}(x) d x= \\
=-\frac{1}{\lambda_{n}^{2}} \psi_{n}^{(2)}-\frac{k}{\lambda_{n}^{2}} \psi_{1 n},
\end{gathered}
$$

где

$$
\psi_{n}^{(2)}=\int_{0}^{l} \psi^{\prime \prime}(x) x^{k} X_{n}(x) d x, \quad \psi_{1 n}=\int_{0}^{l} \frac{\psi^{\prime}(x)}{x} x^{k} X_{n}(x) d x .
$$

Из (47) следует вторая оценка из (41).

Согласно лемме 3, ряды (38)-(40) мажорируются сходящимся числовым рядом

$$
C_{12} \sum_{n=1}^{\infty} n^{-\frac{3}{2}}
$$

а следовательно, ряды $(27)-(29)$ в области $\bar{D}$ по признаку Вейерштрасса сходятся равномерно.

Таким образом, построена функция $u(x, t)$, определяемая рядом $(27)$, которая удовлетворяет всем условиям задачи (2)-(5), (8). Тем самым доказана

Теорема 2. Если функиии $\varphi(x)$ и $\psi(x)$ удовлетворяют условиям леммъ 3 , то существует единственное решение $u(x, t)$ задачи $(2)-(5),(8)$, определяемое рядом (27), при этом $u(x, t) \in C^{2}(\bar{D})$.

Теорема 3. Если функиии $\varphi(x)$ и $\psi(x)$ удовлетворяют условиям леммы 3 и условиям согласования (7), то существует единственное решение задачи (2)-(7), определяемое рядом (27), при этом $u(x, t) \in C^{2}(\bar{D})$.

Д оказ а тель ст во. Пусть $u(x, t)$ - решение задачи $(2)-(5),(8)$ и функции $\varphi(x)$ и $\psi(x)$ удовлетворяют условиям теоремы. Тогда уравнение $(1)$ выполняется всюду в области $D$. Умножим уравнение $(1)$ на $x$ и проинтегрируем при фиксированном $t \in(0, T)$ по переменной $x$ на промежутке от $\varepsilon$ до $l-\varepsilon$, где $\varepsilon>0$ - достаточно малое число. В результате получим

$$
\int_{\varepsilon}^{l-\varepsilon} u_{t t}(x, t) x d x-\left.\left(x u_{x}+(k-1) u\right)\right|_{\varepsilon} ^{l-\varepsilon}=0 .
$$


В последнем равенстве, переходя к пределу при $\varepsilon \rightarrow 0$, с учетом условий (5) и (8) будем иметь

$$
\int_{0}^{l} u_{t t}(x, t) x d x=0 .
$$

Проинтегрируем равенство (48) по переменной $t$, в результате имеем

$$
\int_{0}^{l} u_{t}(x, t) x d x=C_{1}=\text { const. }
$$

Полученное равенство (49) проинтегрируем еще раз по переменной $t$. Тогда справедливо равенство

$$
\int_{0}^{l} u(x, t) x d x=C_{1} t+C_{2}, \quad C_{2}=\text { const. }
$$

Полагая в равенстве (49) $t=0$, с учетом условий (4) и (7) найдем

$$
\int_{0}^{l} u_{t}(x, 0) x d x=\int_{0}^{l} \psi(x) x d x=C_{1}=0 .
$$

Положим теперь $t=0$ в равенстве (50), откуда с учетом условий (4) и (7) и найденного значения $C_{1}=0$ получим

$$
\int_{0}^{l} u(x, 0) x d x=\int_{0}^{l} \varphi(x) x d x=C_{2}=A .
$$

Подставляя найденные значения констант $C_{1}=0$ и $C_{2}=A$ в формулу (50), имеем

$$
\int_{0}^{l} u(x, t) x d x=A .
$$

Тем самым мы показали, что при выполнении условий согласования (7), условия (6) и (8) эквивалентны. Значит, эквивалентны и задачи $(2)-(7)$ и (2)-(5), (8).

Декларация о финансовых и других взаимоотношениях. Исследование не имело спонсорской поддержки. Автор несет полную ответственность за предоставление окончательной версии рукописи в печать. Окончательная версия рукописи была одобрена автором. Автор не получал гонорар за статью.

\section{ORCID}

Наталья Владимировна Зайцева: http://orcid.org/0000-0001-9907-9641

\section{БИБЛИОГРАФИЧЕСКИЙ СПИСОК}

1. Киприянов И. А. Сингулярные эллиптические краевые задачи. М.: Физматлит, 1997. 208 c.

2. Пулькин С. П. Избранные труды. Самара: Универс групп, 2007. 264 с.

3. Пулькин С. П. О единственности решения сингулярной задачи Геллерстедта // Изв. вузов. Матем., 1960. №6. С. 214-225.

4. Сабитов К. Б., Ильясов Р. Р. О некорректности краевых задач для одного класса гиперболических уравнений // Изв. вузов. Матем., 2001. № 5. С. 59-63. 
5. Сабитов К. Б., Ильясов Р. Р. Решение задачи Трикоми для уравнения смешанного типа с сингулярным коэффициентом спектральным методом// Изв. вузов. Матем., 2004. № 2. C. $64-71$.

6. Cannon J. R. The solution of heat equation subject to the specification of energy // Quart. Appl. Math, 1963. vol.21, no. 2. pp. 155-160. doi: 10.1090/qam/160437.

7. Камынин Л. И. Об одной краевой задаче теории теплопроводности с неклассическими граничными условиями // Ж. вычисл. матем. и матем. физ., 1964. Т. 4, №6. С. 10061024.

8. Ионкин Н. И. Решение одной краевой задачи теории теплопроводности с неклассическим краевым условием // Дифферени. уравнения, 1977. Т. 13, № 2. С. 294-304.

9. Пулькина Л. С. Нелокальная задача с интегральными условиями для гиперболического уравнения // Дифферени. уравнения, 2004. Т. 40, № 7. С. 887-892.

10. Пулькина Л. С. Краевые задачи для гиперболического уравнения с нелокальными условиями I и II рода // Изв. вузов. Матем., 2012. № 4. С. 74-83.

11. Пулькина Л. С. Задачи с неклассическими условиями для гиперболических уравнений. Самара: Самарский университет, 2012. 194 с.

12. Бенуар Нур-Эддин, Юрчук Н. И. Смешанная задача с интегральным условием для параболических уравнений с оператором Бесселя // Дифферени. уравнения, 1991. Т. 27, № 12. C. 2094-2098.

13. Beilin S. A. Existence of solutions for one-dimensional wave equations with nonlocal conditions // Electronic Journal of Differential Equations, 2001. vol.2001, no.76. pp. 1-8, http://ejde.math.txstate.edu/Volumes/2001/76/abstr.html.

14. Mesloub S., Bouziani A., Kechkar N. A strong solution of an envolution problem with integral condition// Georgian Mathematical Journal, 2002. vol.9, no.1. pp. 149-159. doi : 10.1515/GMJ.2002.149.

15. Сабитова Ю. К. Нелокальные начально-граничные задачи для вырождающегося гиперболического уравнения // Изв. вузов. Матем., 2009. № 12. С. 49-58.

16. Сабитов К. Б. Краевая задача для уравнения параболо-гиперболического типа с нелокальным интегральным условием // Дифферени. уравнения, 2010. Т. 46, № 10. С. 14681478.

17. Сабитов К. Б. Нелокальная задача для уравнения параболо-гиперболического типа в прямоугольной области // Матем. заметки, 2011. Т. 89, № 4. С. 596-602. doi: 10 . $4213 / \mathrm{mzm} 8462$.

18. Сабитова Ю. К. Краевая задача с нелокальным интегральным условием для уравнений смешанного типа с вырождением на переходной линии // Матем. заметки, 2015. Т. 98, № 3. C. 393-406. doi : 10.4213/mzm9135.

19. Сабитов К. Б. Уравнения математической физики. М.: Физматлит, 2013. 352 с.

20. Zaitseva N. V. Keldysh type problem for $B$-hyperbolic equation with integral boundary value condition of the first kind // Lobachevskii J. Math., 2017. vol. 38, no. 1 (to appear).

21. Олвер Ф. Введение в асимптотические методы и специальные функиии. М.: Мир, 1986. $381 \mathrm{c.}$

22. Сабитов К. Б., Вагапова Э. В. Задача Дирихле для уравнения смешанного типа с двумя линиями вырождения в прямоугольной области // Дифферени. уравнения, 2013. Т. 49, № 1. С. $68-78$.

23. Сафина Р. М. Задача Келдыша для уравнения смешанного типа второго рода с оператором Бесселя // Дифферени. уравнения, 2015. Т. 51, № 10. С. 1354-1366.

Поступила в редакцию 12/VII/2016; в окончательном варианте - 12/XII/2016; принята в печать - 16/IX/2016. 
Vestn. Samar. Gos. Techn. Un-ta. Ser. Fiz.-mat. nauki

[J. Samara State Tech. Univ., Ser. Phys. \& Math. Sci.], 2016, vol. 20, no. 4, pp. 589-602

ISSN: 2310-7081 (online), 1991-8615 (print)

doi: http://dx.doi.org/10.14498/vsgtu1501

MSC: 35L20, 35L81

\title{
THE NONLOCAL PROBLEM FOR A HYPERBOLIC EQUATION WITH BESSEL OPERATOR IN A RECTANGULAR DOMAIN
}

\author{
N. V. Zaitseva \\ Kazan (Volga Region) Federal University, \\ 18, Kremlyovskaya str., Kazan, 420008, Russian Federation.
}

\begin{abstract}
We consider a boundary value problem for a hyperbolic equation with Bessel differential operator in a rectangular domain with integral nonlocal boundary value condition of the first kind. The equivalence between boundary value problem with integral nonlocal condition of the first kind and a local boundary value problem with mixed boundary conditions of the first and third kinds is proved. The existence and uniqueness of solution of the equivalent problem are established by means of the spectral method. At the uniqueness proof the completeness of the eigenfunction system of the spectral problem is used. At the existence proof the assessment of coefficients of series, the asymptotic formula for Bessel function of the first kind and asymptotic formula for eigenvalues are used. Sufficient conditions on the functions defining initial data of the problem are received. The solution of the problem is obtained in explicit form. The solution is obtained in the form of the Fourier-Bessel series. Its convergence is proved in the class of regular solutions.
\end{abstract}

Keywords: hyperbolic equation, singular coefficient, Bessel differential operator, non-local boundary value condition, uniqueness, existence, FourierBessel series, uniform convergence.

Declaration of Financial and Other Relationships. The research has not had any sponsorship. The author is absolutely responsible for submitting the final manuscript in print. The author has approved the final version of manuscript. The author has not received any fee for the article.

\section{ORCID}

Natalya V. Zaitseva: http://orcid.org/0000-0001-9907-9641

(C) 2016 Samara State Technical University.

Please cite this article in press as:

Zaitseva N. V. The nonlocal problem for a hyperbolic equation with Bessel operator in a rectangular domain, Vestn. Samar. Gos. Tekhn. Univ., Ser. Fiz.-Mat. Nauki [J. Samara State Tech. Univ., Ser. Phys. \& Math. Sci.], 2016, vol. 20, no. 4, pp. 589-602. doi: 10.14498/vsgtu1501. (In Russian)

Author Details:

Natalya V. Zaitseva (n.v.zaiceva@yandex.ru) (Assistant), Dept. of Higher Mathematics and Mathematical Modeling. 


\section{REFERENCES}

1. Kipriyanov I. A. Singuliarnye ellipticheskie kraevye zadachi [Singular elliptic boundary value problems]. Moscow, Fizmatlit, 1997, 208 pp. (In Russian)

2. Pul'kin S. P. Izbrannye trudy [Selected works]. Samara, Univers grupp, 2007, 264 pp. (In Russian)

3. Pul'kin S. P. Uniqueness of the solution of a singular problem of Gellerstedt-Tricomi, Izv. Vyssh. Uchebn. Zaved. Mat., 1960, no. 6, pp. 214-225 (In Russian).

4. Sabitov K. B., Il'yasov R. R. On the ill-posedness of boundary value problems for a class of hyperbolic equations, Russian Math. (Iz. VUZ), 2001, vol. 45, no. 5, pp. 56-60.

5. Sabitov K. B., Il'yasov R. R. Solution of the Tricomi problem for an equation of mixed type with a singular coefficient by means of the spectral method, Russian Math. (Iz. VUZ), 2004, vol. 48, no. 2, pp. 61-68.

6. Cannon J. R. The solution of heat equation subject to the specification of energy, Quart. Appl. Math, 1963, vol. 21, no. 2, pp. 155-160. doi: 10.1090/qam/160437.

7. Kamynin L. I. A boundary value problem in the theory of heat conduction with a nonclassical boundary condition, U.S.S.R. Comput. Math. Math. Phys., 1964, vol. 4, no. 6, pp. 33-59. doi : 10.1016/0041-5553(64)90080-1.

8. Ionkin N. I. The solution of a certain boundary value problem of the theory of heat conduction with a nonclassical boundary condition, Differ. Uravn., 1977, vol. 13, no. 2, pp. 294-304 (In Russian).

9. Pul'kina L. S. A Nonlocal Problem with Integral Conditions for a Hyperbolic Equation, Differ. Equ., 2004, vol.40, no. 7, pp. 947-953. doi: 10.1023/B:DIEQ.0000047025.64101.16.

10. Pul'kina L. S. Boundary-value problems for a hyperbolic equation with nonlocal conditions of the I and II kind, Russian Math. (Iz. VUZ), 2012, vol. 56, no. 4, pp. 62-69. doi: 10.3103/ S1066369X12040081.

11. Pul'kina L. S. Zadachi s neklassicheskimi usloviiami dlia giperbolicheskikh uravnenii [Problems with Nonclassical Conditions for Hyperbolic Equations]. Samara, Samara Univ., 2012, 194 pp. (In Russian)

12. Benuar Nur-Eddin, Yurchuk N. I. A mixed problem with integral condition for parabolic equations with a Bessel operator, Differ. Equ., 1991, vol. 27, no. 12, pp. 1482-1487.

13. Beilin S. A. Existence of solutions for one-dimensional wave equations with nonlocal conditions, Electronic Journal of Differential Equations, 2001, vol.2001, no.76, pp. 1-8, http://ejde.math.txstate.edu/Volumes/2001/76/abstr.html.

14. Mesloub S., Bouziani A., Kechkar N. A strong solution of an envolution problem with integral condition, Georgian Mathematical Journal, 2002, vol. 9, no. 1, pp. 149-159. doi: 10. 1515/GMJ . 2002.149.

15. Sabitova Yu. K. Nonlocal initial-boundary-value problems for a degenerate hyperbolic equation, Russian Math. (Iz. VUZ), 2009, vol.53, no.12, pp. 41-49. doi:10.3103/ S1066369X09120068.

16. Sabitov K. B. Boundary value problem for a parabolic-hyperbolic equation with a nonlocal integral condition, Differ. Equ., 2010, vol.46, no.10, pp. 1472-1481. doi: 10.1134/ S0012266110100113.

17. Sabitov K. B. Nonlocal problem for a parabolic-hyperbolic equation in a rectangular domain, Math. Notes, 2011, vol.89, no.4, pp. 562-567. doi: 10.1134/S0001434611030278.

18. Sabitova Yu. K. Boundary-value problem with nonlocal integral condition for mixed-type equations with degeneracy on the transition line, Math. Notes, 2015, vol. 98, no. 3, pp. 454465. doi: 10.1134/S0001434615090114.

19. Sabitov K. B. Uravneniia matematicheskoi fiziki [Equations of mathematical physics]. Moscow, Fizmatlit, 2013, 352 pp. (In Russian)

20. Zaitseva N. V. Keldysh type problem for $B$-hyperbolic equation with integral boundary value condition of the first kind, Lobachevskii J. Math., 2017, vol. 38, no. 1 (to appear).

21. Olver F. W.J. Asymptotics and special functions. Reprint. Wellesley, MA, A K Peters, 1997, $\mathrm{xviii}+572 \mathrm{pp}$. 
22. Sabitov K. B., Vagapova E. V. Dirichlet problem for an equation of mixed type with two degeneration lines in a rectangular domain, Differ. Equ., 2013, vol.49, no. 1, pp. 68-78. doi : 10.1134/S0012266113010072.

23. Safina R. M. Keldysh problem for a mixed-type equation of the second kind with the Bessel operator, Differ. Equ., 2015, vol.51, no.10, pp. 1347-1359. doi:10.1134/ S0012266115100109.

Received 12/VII/2016;

received in revised form $12 / \mathrm{XII} / 2016$;

accepted 16/IX/2016. 